\title{
Entre desafios e possibilidades: \\ estratégias para ensinar a cuidar em \\ enfermagem do adolescente com câncer*
}

\author{
BETWEEN CHALLENGES AND POSSIBILITIES: STRATEGIES FOR TEACHING CARE \\ NURSING FOR ADOLESCENTS WITH CANCER
}

\author{
ENTRE DESAFÍOS Y POSIBILIDADES: ESTRATEGIAS PARA ENSEÑAR A CUIDAR \\ EN ENFERMERÍA DELADOLESCENTE CON CÁNCER
}

Sônia Regina de Souza', Isabel Cristina dos Santos Oliveira²

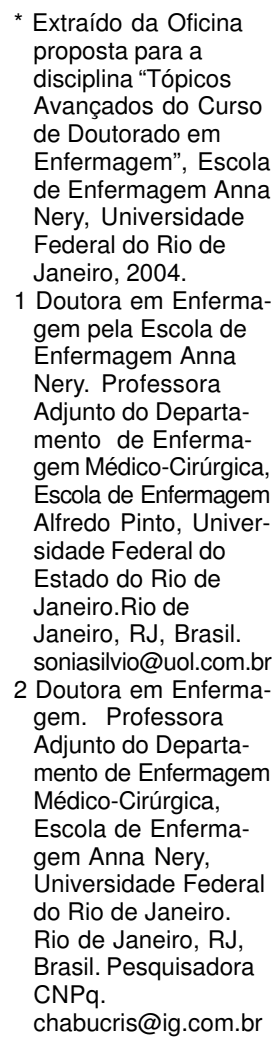

1 Doutora em Enfermagem pela Escola de Enfermagem Anna

chabucris@ig.com.br

\begin{abstract}
RESUMO
Trata-se de um relato de experiência, que tem como objetivo apresentar reflexões acerca das experiências vivenciadas junto aos alunos do Curso de Graduação em Enfermagem da Escola de Enfermagem Alfredo Pinto da Universidade Federal do Estado do Rio de Janeiro (UNIRIO). No cotidiano da prática de ensino, os alunos se deparam com adolescentes acometidos pelos distúrbios onco-hematológicos. Constatou-se que, para os estudantes, era um desafio aprender a cuidar do adolescente em um momento de adoecimento pelo câncer, doença que traz importantes significados no imaginário social. Como estratégia para a aprendizagem, optou-se por uma atividade denominada oficina de idéias, a qual possibilitava aos alunos que expressassem seus sentimentos, idéias (pré) concebidas e expectativas acerca do cuidar do adolescente com câncer. Como resultados, evidenciou-se um singular momento de reflexão/sensibilização que resulta em um preparo e um reconhecimento das peculiaridades desse grupo e atitude de aproximação para o cuidar no espaço hospitalar.
\end{abstract}

\section{DESCRITORES}

Educação em enfermagem.

Enfermagem oncológica.

Adolescente.

Oncologia.

Aprendizagem.

\section{ABSTRACT}

This is an experience report that has the goal of presenting reflections on the experiences lived with undergraduate Nursing students of the Federal University of the State of Rio de Janeiro. In the dayto-day teaching practice students have to deal with adolescents that are victims of onco-hematogenic disorders. It was observed that for the students it was a challenge to learn how to care for adolescents suffering from cancer, an illness that brings with it important significances in the social imaginary. As a learning strategy was used an activity called workshop of ideas, which made possible for the students to express their feelings, their (pre)conceived ideas and their expectations regarding the care for adolescents with cancer. Results evidenced a unique moment of reflection/sensibilization that leads to a preparation and to recognition of the peculiarities of this group of patients, and to an attitude that brings them closer to caring within the hospital space.

\section{RESUMEN}

Se trata de un relato de experiencia, que tiene como objetivo presentar reflexiones sobre las experiencias vividas junto a los alumnos del Curso de Graduación en Enfermería de la Universidad Federal do Estado de Rio de Janeiro (UNIRIO). En el cotidiano de la práctica de enseñanza, los alumnos se deparan con adolescentes acometidos por los disturbios oncológicos-hematológicos. Se constató que para los alumnos el desafío era aprender a cuidar al adolescente en el momento del cáncer. Como estrategia para el aprendizaje, se optó por una actividad denominada Oficina de Ideas. Esta posibilitó que los alumnos expresasen sus sentimientos y expectativas de como cuidar al adolescente con cáncer. Como resultado se evidenció un singular momento de reflexión que resulta en un reconocimiento de las peculiaridades de este grupo y actitudes de aproximación para el cuidar en el espacio hospitalario.

\section{KEY WORDS}

Education, nursing.

Oncologic nursing.

Adolescent.

Medical oncology.

Learning.

\section{DESCRIPTORES}

Educación en enfermería.

Enfermería oncológica.

Adolescente.

Oncología médica.

Aprendizaje. 


\section{INTRODUÇÃO}

No cotidiano da prática de ensinar a cuidar em enfermagem no espaço hospitalar, muitos desafios se fazem presentes. Esses desafios englobam desde a existência ou escassez de recursos materiais e tecnológicos à capacitação qualitativa e quantitativa de recursos humanos.

Por outro lado, para o aluno de enfermagem, que inicia seu processo de aprendizagem na perspectiva de cuidar de clientes que se apresentam adoecidos, outro desafio se faz premente, o estabelecimento de um vínculo, de uma relação de empatia. Tal condição configura-se em uma etapa crucial para que se efetive a aprendizagem. Durante o desenvolvimento das atividades teóricas-práticas foi possível observar que os alunos de enfermagem mostravam-se surpresos, quando se deparavam com os adolescentes, em sua maioria, com leucemia e linfoma, que estavam na faixa etária diferente daquela que comumente se encontra em uma unidade de internação de pessoas portadoras de doenças crônicas.

Na qualidade de docente na disciplina Cuidar/Cuidado em Oncologia do Curso de Graduação em Enfermagem da Escola de Enfermagem Alfredo Pinto da Universidade Federal do Estado do Rio de Janeiro (EEAP/UNIRIO) e doutoranda da Escola de Enfermagem Anna Nery da Universidade Federal do Rio de Janeiro (EEAN/UFRJ), na Linha de Pesquisa Enfermagem Hospitalar, que tem como objeto de sua tese o cotidiano das enfermeiras junto aos adolescentes com distúrbio oncohematológico, vislumbrou-se a possibilidade de planejar uma atividade junto aos alunos de enfermagem que enfocasse os referidos adolescentes considerando que o tema adolescente hospitalizado é pouco discutido durante o curso de graduação em enfermagem.

\section{O ADOLESCENTE EO ALUNO DE ENFERMAGEM: ENCONTROSNO CENÁRIOHOSPITALAR}

A Organização Mundial da Saúde (OMS), desde 1989, reconhece a adolescência como um momento de importância vital e define esse período da vida humana como um processo fundamentalmente biológico de vivências orgânicas, no qual se aceleram o desenvolvimento cognitivo e a estruturação da personalidade.

A OMS apresenta parâmetros para esta fase da vida humana construídos socialmente, culturalmente e biologicamente. Essas demarcações vão desde as relacionadas a faixa etária (pré-adolescência -10 a 14 anos e a adolescência propriamente dita -15 aos 19 anos) até a um entendimento mais complexo considerando o desenvolvimento cognitivo e a própria personalidade do adolescente.

Em 1988, a Constituição Federal no Brasil, efetiva como dever do Estado e da Sociedade Civil, a garantia do atendi- mento às necessidades em saúde das crianças e dos adolescentes. O Programa de Saúde do adolescente - PROSAD do Ministério da Saúde que traça as normas para a implementação nacional de uma atenção integral à saúde do adolescente, estabelece a faixa etária que vai dos 10 aos 19 anos para a adolescência ${ }^{(1)}$.

Em pesquisa realizada com Instituições que atendem a adolescentes em São Paulo, constatou-se que na área hospitalar não existem leitos próprios para adolescentes, sendo os mesmos internados em momentos na clínica médica como adultos, em outros momentos na pediatria, como crianças ${ }^{(2)}$.

De acordo com a Resolução n ${ }^{\circ}$ 41, de 13 de outubro de 1995, do Conselho Nacional dos Direitos da Criança e do Adolescente (CONANDA) que trata dos direitos da criança e dos adolescentes hospitalizados, são vinte os direitos assegurados a eles na qual se destaca, entre outros, o direito de desfrutar de alguma forma de recreação, programa de educação para a saúde, acompanhamento do currículo escolar durante sua permanência hospitalar ${ }^{(3)}$.

Constata-se, porém que inúmeros serviços de saúde encontram-se despreparados para atender ao adolescente, para atenção às suas peculiaridades e a complexidade de suas necessidades.

Segundo o Instituto Nacional do Câncer (INCA) excluindose as causas mal definidas, o câncer constitui a segunda causa de morte no Brasil por doença, atrás somente das doenças cardiovasculares ${ }^{(4)}$. Seguindo um raciocínio clínico que orienta os programas de prevenção e controle do câncer no Brasil, o INCA apresenta como fatores determinantes sociais do câncer a urbanização, a industrialização e a maior expectativa de vida. Tais fatores contribuem para o aumento de agente cancerígena ambiental ou para uma maior e mais prolongada exposição dos seres humanos a estes agentes.

No que diz respeito à epidemiologia dos tumores da criança e do adolescente, de acordo com o INCA, tem se verificado um aumento progressivo das taxas de incidência na criança e no adolescente, com destaque respectivamente para a leucemia linfocítica aguda (LLA), os tumores do sistema nervoso central, os linfomas não-hodgkin e os tumores renais ${ }^{(5)}$. Em contrapartida, também se observa um aumento linear nas taxas de cura (70 a $80 \%)$ dos casos dos tumores na infância.

Para o aluno de enfermagem, observa-se que o lidar com a realidade concreta de relacionar-se com o adolescente acometido pelo distúrbio oncohematológico representa reavaliar sua própria adolescência, que na maioria das vezes, encontra-se muito próxima.

Vale destacar que dependendo da patologia que o adolescente for acometido, o mesmo poderá apresentar-se em 
fase de completa recuperação ou nas doenças mais graves, apresentar-se vivenciando o processo de morrer e todos os seus aspectos inter-relacionados. Nesse sentido, para o aluno de enfermagem configura-se em um verdadeiro convite para um repensar a própria vida e o sentimento de imortalidade que comumente acompanha o adolescente.

$\mathrm{O}$ atendimento ao adolescente representa um desafio às qualidades e habilidades do profissional, requerendo destes além do conhecimento técnico-científico, disponibilidade, flexibilidade, capacidade de interação, reconhecimento de limites e posturas éticas que assegurem a confidência e segurança, que são indispensáveis para o sucesso no relacionamento com o adolescente.

A equipe multiprofissional ao prestar qualquer tipo de assistência, direta ou indiretamente ao adolescente deve ter bem claro a enorme multiplicidade de fatores que influenciam a vida do adolescente. Com isto

\begin{abstract}
é necessário somar as variáveis pessoais, o que faz de cada adolescente um ser único, como são, todos os seres humanos em qualquer fase da sua vida. Por isso mesmo, generalizações sobre as necessidades ou sobre as condutas dos adolescentes devem se vistas com muitas restrições, pois não há atributos absolutos para a adolescência(6).
\end{abstract}

O adoecimento pelo distúrbio oncohematológico na adolescência constitui um fenômeno caracterizado por um acontecimento, que comporta uma série de modificações de ordem objetiva, e subjetiva que pode representar para o adolescente um não ser igual. Neste sentido, com as lentes do hospital o adolescente passa de uma condição de saudável ou normal para portador de uma doença ou anomalia reconhecida e legitimada por um diagnóstico médico.

A hospitalização do adolescente gera mudanças dolorosas em todos os aspectos de sua vida. O impacto do diagnóstico para o adolescente e sua família constitui uma temática, que deve ser considerada quando aproxima - se da realidade de cuidar deste grupo no cenário hospitalar. $\mathrm{O}$ diagnóstico médico é, ao mesmo tempo, esperado e temido pelo adolescente e sua família. São situações novas e difíceis, nas quais o número de informações é grande e seu conteúdo é, por vezes, doloroso ${ }^{(7)}$.

Assim, os alunos de enfermagem se deparam com a complexidade do adoecer e as consequiências do tratamento dos distúrbios onco-hematológicos que acometem os adolescentes. Neste encontro, surge o desafio de reconhecer e considerar esse adolescente em sua totalidade. È importante aclarar a visão que se tem do adolescente, que pode gerar condutas inadequadas e resultados negativos ${ }^{(6)}$.

O contato inicial gera a chamada primeira impressão, o impacto que cada um causa ao outro. Essa primeira impressão está condicionada a um conjunto de fatores psicológicos da experiência anterior de cada pessoa, suas expectati- vas e motivação no momento e a própria situação do encontro ${ }^{(8)}$. Quando uma pessoa começa a participar de um grupo, há uma base interna de diferenças que englobam conhecimentos, informações, opiniões, preconceitos, atitudes, gostos, crenças e valores, que resultam em diferentes estilos comportamentais e traz diferenças de percepções e sentimentos relacionados à situação compartilhada ${ }^{(8)}$.

No decorrer do cuidado prestado às pessoas que se encontram em situação fragilizada, dependente é desejável que o aluno de enfermagem esteja dotado de maior grau de sensibilidade. Esta condição pessoal favorece o reconhecimento das necessidades subjetivas do adolescente ${ }^{(9)}$.

A atitude de cuidado surge quando a existência de alguém tem importância para o outro, o que resulta em dedicação, disponibilidade, pré (ocupação) e responsabilidade de quem cuida para aquele que é cuidado. Eis o desafio: diante da diversidade de experiências e expectativas dos alunos em relação ao adolescente frente à realidade concreta de aprender a cuidar do adolescente hospitalizado com distúrbio oncohe-matológico no espaço hospitalar existe uma lacuna. Essa lacuna é reflexo da carência de pesquisas sobre o adoecimento na adolescência, e de uma prática profissional dicotomizada e ainda centrada no modelo biológico ${ }^{(10)}$.

Sendo assim, faz-se necessário a utilização de alternativas no processo de ensino-aprendizagem. Essas práticas significam uma ruptura com a rotina educativa caracterizada muitas vezes pela fragmentação e pelo tecnicismo. Acredito que essas questões devem ser discutidas no momento em que o aluno as vivencia, pois na medida em que ele se compreende nesse processo, estará capacitando-se, além de tornar-se mais comprometido com o cuidar a esses adolescentes ${ }^{(11)}$.

Entende-se que ousar no espaço da sala de aula é reconhecer esse espaço como propício para descobertas, um lugar onde as atitudes podem e devem ser discutidas. Optou-se, portanto por uma prática alternativa no processo de ensino - aprendizagem denominada oficina de idéias. Como docente e facilitadora da oficina meu papel foi o de mediadora das opiniões, apreendendo as temáticas que emergiam e organizando o processo de desenvolvimento de idéias apresentadas pelos alunos.

\section{APRENDER A CUIDAR DE ADOLESCENTES COM DISTÚRBIO ONCO-HEMATOLÓGICO NO ESPAÇO HOSPITALAR: OFICINA DE IDÉIAS}

A Metodologia Participativa é aquela que permite a atuação do aluno de enfermagem, valorizando seus conhecimentos e experiências, convidando-os a discussão e principalmente instrumentalizá-lo a fim de buscar soluções para os problemas que emergem do cotidiano no processo ensino-aprendizagem. Trata-se de um caminho didático-pedagógico que considera a experiência e a participação do alu- 
no a partir de situações reais que podem ser abordadas de forma lúdica.

O momento de se fazer uma proposta lúdica é muito importante. Princípios devem ser seguidos como, por exemplo, o número de participantes, o tempo de socialização dos sentimentos e descobertas e, principalmente, ter claro o eixo temático que deverá ser tratado pelo grupo ${ }^{(12)}$.

\section{Momento um: acolhendo os alunos}

No dia 30 de abril de 2004 no horário de 13h30min às 17 horas na sala 305 aconteceu a $1^{\text {a }}$ Oficina para apresentar junto aos alunos de Enfermagem a proposta da Oficina de Idéias.

Às $13 \mathrm{~h} 30 \mathrm{~min}$ foi realizado o acolhimento dos alunos com música ambiente. Acolher significa mobilizar afetos. Esse momento diz respeito a um entrosamento, um momento de descontração, no qual haja possibilidade de aproximação dos participantes, pelo toque ou por movimentos e cantos $^{(13-14)}$.

Os alunos foram convidados a uma atividade de integração onde cantaram a música "Tempo Perdido" de autor desconhecido interpretada pelo grupo musical Legião Urbana. Seguem trechos da letra da música:

Todos os dias quando acordo/Não tenho mais o tempo que passou Mas tenho muito tempo/Temos todo o tempo do mundo.Todos os dias antes de dormir/Lembro e esqueço como foi o dia: Sempre em frente/Não temos tempo a perder.Temos nosso próprio tempo/Somos tão jovens.

O objetivo dessa atividade era 'quebrar o gelo inicial' com uma música popular e que falava de tempo e juventude, acolhendo os alunos para os momentos seguintes da oficina. Após a atividade de integração, os alunos se acomodaram em círculo e foi apresentada a proposta da Oficina de idéias.

Momento dois: idéias, idéias, idéias - uma tempestade!

Às 14 horas iniciou-se uma atividade denominada brainstorming ou tempestade de déias. A palavra idéia pode ser empregada como um objeto qualquer do pensamento humano, ou seja, como uma representação em geral ${ }^{(15)}$.

Essa atividade é concebida para incentivar a livre promoção de idéias, sem restrições nem limitações. Funciona como um mecanismo de indução, que desenvolve do ponto de vista pedagógico, uma atitude interrogativa e reflexiva diante de um dado questionamento ${ }^{(14) .}$ Para tal foram escrito no quadro-negro as palavras adolescente e adolescência. Solicitou-se aos alunos que representassem por meio de palavras as idéias que tinham acerca desses termos. Os relatos foram diversos, conforme o trecho a seguir,

Rebelde; alguém que gosta de música alta; trancar-se no quarto; andar em grupo; sente-se incompreendido; não obe- dece a regras; difícil; fase de transformações; mudanças no corpo; difícil comunicação; desejo de ser aceito no grupo.

Constatou-se que essa atividade permitiu que os alunos tivessem contato com os conceitos internalizados, aquilo que está no imaginário de cada um a respeito da adolescência e do adolescente. Nesse instante relembraram sua própria adolescência caracterizando a mesma como um período complicado. Essa atividade teve aproximadamente trinta minutos de duração.

Após a tempestade de idéias foi solicitado aos alunos que representassem o adolescente em uma condição diferenciada - adoecido pelo distúrbio oncohematológico. Essa atividade é entendida como atividade de desafio e ou de reflexão ${ }^{(14)}$. Os alunos foram subdivididos em cinco grupos e com o auxílio de lápis de cera e folhas de papel tipo pardo, representaram como entendiam o adolescente adoecido hospitalizado com distúrbio oncohematológico e como deveria ser o espaço no hospital para cuidar desse adolescente.

Essa atividade teve duração de 40 minutos e os alunos se mostraram motivados. Foi possível observar que, durante o desenvolvimento, os alunos inicialmente discutiam entre si sobre o que cada um acreditava ser a representação desse adolescente na condição de hospitalizado e logo após buscavam uma forma de representação que atendesse às expectativas do grupo.

As atividades retornaram às $15 \mathrm{~h} 20 \mathrm{~min}, \operatorname{logo}$ após um intervalo para um lanche, com os alunos apresentando suas produções estéticas ao restante do grupo.

$\mathrm{O}$ adolescente hospitalizado foi representado pelos alunos como estando triste, sozinho e isolado, em uma estrada com dois caminhos, que pode levar a cura ou não dependendo da aceitação da doença pelo mesmo; como estando em um túnel, túnel da vida e que pela frente ele encontra uma porta dizendo que é proibido ultrapassar; que está com medo, dor, desejo, sofrimento, desespero, angústia, sofrimento, morte e vazio.

Quanto às produções estéticas acerca do ambiente para o cuidado desses adolescentes, os alunos apresentaram um ambiente em que o adolescente se sentisse acolhido, com outros adolescentes, familiares e pessoas significativas. Apontaram para a necessidade do ambiente ser terapêutico contendo objetos que fossem comuns na vida do adolescente como, por exemplo, o computador, o rádio e que fosse o mais próximo daquele vivido pelo adolescente, por exemplo, em casa e que o ambiente deveria também significar para o adolescente um local de esperança, uma luz no fim do túnel.

Os alunos relataram que a enfermagem pode ajudar ao adolescente nesse momento fazendo-o acreditar na vida durante a doença/câncer. Essa fase da atividade durou uma hora e dez minutos e a participação foi intensa. Muitos alunos debateram e emitiram opiniões acerca das produções dos colegas. 
Logo após elaborou-se uma síntese daquilo que havia sido discutido, o que os alunos pensam saber sobre a adolescência e as idéias que estavam no imaginário sobre esse adolescente com câncer. Destacaram-se as formas como os alunos representavam um ambiente próximo ao ideal para cuidar dos adolescentes. Acredita-se que valorizar a subjetividade da experiência particular, do simbólico e da criatividade do aluno permite reconhecer os limites e as possibilidades frente a uma dada situação da realidade concreta.

\section{Momento três: a despedida}

Ao término da oficina, foi feito uma breve avaliação, com depoimentos espontâneos dos alunos, que comentaram a importância da oficina, o despertar do interesse pelos adolescentes. Alguns expressaram, em suas falas, como tiveram contato com os adolescentes no hospital, e das dificuldades vivenciadas de aproximação, pois eles não esperavam encontrar adolescentes junto com os adultos e muito menos adoecidos pelo câncer.

O último momento da oficina de idéias foi exatamente da forma como iniciamos: cantando uma música de autoria de Dado Villa-Lobos/Renato Russo/Marcelo Bonfá de interpretada pelo grupo musical Legião Urbana. O intuito já não era 'quebrar o gelo inicial' e, sim, nos despedirmos cantando a possibilidade de um outro olhar sobre o cuidado ao adolescente que passa pelo reconhecimento das pe-

\section{REFERÊNCIAS}

1. Brasil. Ministério da Saúde. Secretaria Executiva. Subsecretaria de Assuntos Administrativos. Coordenação Geral de Documentação e Informação. Estatuto da criança e do adolescente. $2^{\mathrm{a}}$ ed. Brasília: Ed. MS; 1997.

2. Cano MAT, Ferriani MGC, Alves AC, Nakata CY. A produção do conhecimento sobre adolescência na enfermagem: período 1983 a 1996. Rev Lat Am Enferm. 1998;6(1):91-7.

3. Conselho Nacional dos Direitos da Criança e do Adolescente (CONANDA). Resolução n. 41, de 13 de outubro de 1995. Dispõe sobre os direitos da criança e do adolescente hospitalizados. Diário Oficial da União, Brasília, 17 out. 1995. Seção 1, p. 16319-20.

4. Brasil. Ministério da Saúde. Instituto Nacional do Câncer (INCA). Ações de enfermagem para o controle do câncer: uma proposta de integração ensino-serviço. $2^{\mathrm{a}}$ ed. Rio de Janeiro; 2002.

5. Brasil. Ministério da Saúde. Instituto Nacional do Câncer (INCA). Estimativas da incidência e mortalidade por câncer. Rio de Janeiro; 2002.

6. Vittiello N. Reprodução e sexualidade: um manual para educadores. São Paulo: CEICH; 1994.

7. Guzman CR, Cano MAT. O adolescente e a hospitalização. Rev Eletrônica Enferm [periódico na Internet]. 2000[citado 2005 out. 25];2(2):[cerca de 10 p.]. Disponível em: http: www.fen.ufg. br/revista/revista2_2/ado_hosp.htm culiaridades dessa fase da vida humana. Seguem trechos da letra da música:

Quando o sol bater/Na janela do teu quarto Lembra e vê/ Que o caminho é um só. Porque esperar se podemos começar tudo de novo

\section{CONSIDERAÇÕES FINAIS... IDÉIAS SEMINAIS}

AOficina de Idéias foi de importância ímpar, na medida em que diferentes olhares se voltaram para uma mesma situação. Acredita-se que este tipo de estratégia facilita e amplia o espectro das ações de Enfermagem, acrescentando atitudes como disponibilidade, flexibilidade, capacidade de interação e reconhecimento das peculiaridades dos adolescentes com distúrbio oncohematológico às habilidades técnicas-científicas necessárias à formação profissional do enfermeiro.

É preciso romper com (pré) conceitos que emergem com uma práxis ritual no mundo da razão técnica instrumental, que distancia do fomento às alternativas voltadas a uma práxis emancipatória ${ }^{(15)}$.

Desta forma a realização da oficina constituiu-se em uma possibilidade concreta de estar trazendo a temática e de repensar o processo de aprender a cuidar, considerando os próprios valores dos alunos de enfermagem.

8. Moscovici F. Desenvolvimento interpessoal. Rio de Janeiro: José Olympio; 2003.

9. Hoga LAK. A dimensão subjetiva do profissional na humanização da assistência à saúde: uma reflexão. Rev Esc Enferm USP. 2004;38(1):13-20.

10. Boff L. Saber cuidar: ética do humano, compaixão pela terra. Petrópolis: Vozes; 1999.

11. Lucarelli E. Ensinar e aprender: sujeitos saberes e pesquisa. Rio de Janeiro: DP\&A; 2000.

12. Gonçalves AM. Dinâmica de grupos na formação de lideranças. Rio de Janeiro: DP\&A; 1998.

13.Sobral V, Tavares CM, Silveira MF. Acolhimento como instrumento terapêutico. In: Santos I, editor. Enfermagem assistencial no ambiente hospitalar: realidade, questões e soluções. São Paulo: Atheneu; 2004. p. 65-70.

14. Minicucci A. Técnicas de trabalho de grupo. São Paulo: Atlas; 1987.

15. Abbagnano N. Dicionário de filosofia. São Paulo: Martins Fontes; 2000. 